\title{
Combination of Mycobacterium tuberculosis RS ratio and CFU improves the ability of murine efficacy experiments to distinguish between drug treatments
}

Christian Dide-Agossou, ${ }^{1 \dagger}$ Allison A. Bauman, ${ }^{2}$ Michelle E. Ramey, ${ }^{2}$ Karen Rossmassler,${ }^{3,4}$ Reem Al Mubarak, ${ }^{3,4}$ Samantha Pauly, ${ }^{3,4}$ Martin I. Voskuil, ${ }^{5,6}$ Maria Garcia-Cremades, ${ }^{7,8}$ Rada M. Savic, ${ }^{6,7,9,10}$

1. Department of Epidemiology, Colorado School of Public Health, Aurora, CO, USA

2. Mycobacteria Research Laboratories, Department of Microbiology, Immunology, and Pathology, Colorado State University, Fort Collins, CO, USA

3. Rocky Mountain Regional VA Medical Center, Aurora, CO, USA

4. Division of Pulmonary Sciences and Critical Care Medicine, University of Colorado Anschutz Medical Campus, Aurora, CO, USA

5. Department of Immunology and Microbiology, University of Colorado Anschutz Medical Campus, Aurora, CO, USA

6. Consortium for Applied Microbial Metrics, Aurora, CO, USA

7. Department of Bioengineering and Therapeutic Sciences, University of California San Francisco, San Francisco, CA, USA

8. Departamento de Farmacia Galénica y Tecnología Alimentaria, Facultad de Farmacia, Universidad Complutense de Madrid, Madrid, Spain

9. Division of Pulmonary and Critical Care Medicine, University of California San Francisco, San Francisco, CA, USA

10. Division of HIV, Infectious Diseases and Global Medicine, University of California San Francisco, San Francisco, CA, USA

11. UCSF Center for Tuberculosis, San Francisco, CA, USA

12. Division of Biostatistics and Bioinformatics, National Jewish Health, Denver, CO, USA

13. Center for Tuberculosis Research, Johns Hopkins University, Baltimore, MD, USA 


\section{ABSTRACT}

50 Murine tuberculosis drug efficacy studies have historically monitored bacterial burden based on

51 colony forming units of $M$. tuberculosis in lung homogenate. In an alternative approach, a

52 recently described molecular pharmacodynamic marker called the RS ratio quantifies drug effect

53 on a fundamental cellular process: ongoing ribosomal RNA synthesis. Here we evaluated the

54 ability of different pharmacodynamic markers to distinguish between treatments in three

$55 \mathrm{BALB} / \mathrm{c}$ mouse experiments at two institutions. We confirmed that different pharmacodynamic

56 markers measure distinct biological responses. We found that a combination of

57 pharmacodynamic markers distinguishes between treatments better than any single marker. The

58 combination of the RS ratio with colony forming units showed the greatest ability to recapitulate

59 the rank order of regimen treatment-shortening activity, providing proof of concept that

60 simultaneous assessment of pharmacodynamic markers measuring different properties will

61 enhance insight gained from animal models and accelerate development of new combination

62 regimens. These results suggest potential for a new era in which antimicrobial therapies are

63 evaluated not only on culture-based measures of bacterial burden but also on molecular assays

64 that indicate how drugs impact the physiological state of the pathogen. 


\section{INTRODUCTION}

There is an urgent need for shorter treatment regimens for both drug-susceptible and

68 drug-resistant tuberculosis (TB). Murine models have historically been the backbone of

69 preclinical evaluation of TB drugs and treatment regimens (1-3). Pharmacodynamic (PD)

70 monitoring in murine drug experiments conventionally measures colony-forming units (CFU) in

71 lung homogenate. Measurement of 16S rRNA burden has been proposed as an alternative

72 measure of Mycobacterium tuberculosis (Mtb) burden (4-6). Importantly, neither change in CFU

73 nor rRNA burden during the period that mice are administered treatment accurately indicates the

74 treatment-shortening activity of drugs or regimens $(1,6,7)$. Therefore, experiments evaluating

75 the efficacy of multidrug regimens are commonly based on the proportion of mice with

76 microbiologic relapse 12 weeks or more after treatment cessation $(8,9)$. Because determination

77 of the relapse proportion requires large, resource-intensive mouse experiments sometimes lasting

78 nine to 10 months, the current standard experimental design is a critical bottleneck in TB

79 regimen evaluation. To accelerate regimen evaluation, there is a need for a PD marker or

80 combination of PD markers that indicate the treatment-shortening activity in shorter, less

81 resource-intensive murine experiments without the need for determination of relapse.

82 We recently proposed a novel molecular PD marker called the RS ratio (10). The RS

83 Ratio measures ongoing ribosomal RNA (rRNA) synthesis in $M t b$ by quantifying the abundance

84 of $M t b$ precursor rRNA (pre-rRNA) relative to stable 23S rRNA. Unlike CFU, 16S rRNA burden

85 and other existing PD markers that enumerate the abundance of $M t b$, the RS Ratio measures the

86 degree to which drugs and regimens interrupt rRNA synthesis. In the absence of drug treatment,

87 RS Ratio was validated as a surrogate for bacterial replication rate (10). In the presence of drug 
treatment, RS ratio differentiates individual drug or drug regimen effect in vitro and in vivo and as such, may represent an important new PD marker (10).

In the current work, we used results from three BALB/c mouse experiments to investigate whether three different PD markers (the RS Ratio, CFU, and 16S rRNA burden) provide the same information or measure different biological responses. We asked whether a combination of PD markers measuring different responses distinguishes between treatments better than any single PD marker. Finally, we evaluated the ability of different PD markers and combinations of markers to indicate the treatment-shortening activity of regimens.

\section{METHODS}

We compared three PD markers in three BALB/c mouse experiments in two labs. Experiment 1 evaluated individual drugs to determine whether the RS Ratio, CFU/lung and 16S rRNA burden measure the same or different biological responses. Experiments 2 and 3 evaluated combination regimens to determine whether change in PD markers during the first weeks of treatment distinguishes between regimens and indicates regimen treatment-shortening activity. Efficacy outcomes from Experiments 2 and 3 are reported elsewhere $(10,11)$.

\section{Description of BALB/c mouse experiments}

Full details of murine protocols are included in Supplemental Information and in other publications $(10,11)$. Briefly, all three experiments employed female pathogen-free BALB/c mice infected by the same high dose aerosol (HDA) procedure in a Glas-Col inhalation exposure system $(12,13)$ and treated mice 5 of 7 days a week via oral gavage. Experiments 1 and 3 were conducted at Colorado State University (CSU) using the Mtb Erdman strain. Experiment 2 was conducted at Johns Hopkins University using the Mtb H37Rv strain. 


\section{Experiment 1: Individual drug treatments in BALB/c mouse HDA infection model}

Treatment began on day 11 and continued for 4 weeks with: bedaquiline $(25 \mathrm{mg} / \mathrm{kg})$, ethambutol (100 mg/kg), isoniazid (25 mg/kg), pyrazinamide (150 mg/kg), rifampin (10 mg/kg) or streptomycin $(200 \mathrm{mg} / \mathrm{kg})$. Each treatment group had eight mice except for the untreated control which had five mice.

\section{Experiments 2 and 3: Multidrug treatments in BALB/c HDA relapsing mouse model}

Experiments 2 and 3 used the standard conventional relapsing mouse model described in Supplemental Material $(1,10,11)$. In Experiment 2, treatment began on day 14 post-infection with: isoniazid, rifampin, pyrazinamide, ethambutol - (HRZE), rifapentine, moxifloxacin, pyrazinamide - (PMZ), bedaquiline, moxifloxacin, pyrazinamide - (BMZ), or bedaquiline, moxifloxacin, pyrazinamide, rifabutin - (BMZRb). In Experiment 3, treatment began on day 11 with: HRZE using doses identical to Experiment 2, pretomanid, moxifloxacin, pyrazinamide (PaMZ), bedaquiline, pretomanid, linezolid - $(\mathrm{BPaL})$, or bedaquiline, pretomanid, moxifloxacin, pyrazinamide (BPaMZ). The doses (in $\mathrm{mg} / \mathrm{kg}$ indicated in subscripts) tested were $\mathrm{H}_{10}, \mathrm{R}_{10}, \mathrm{Z}_{150}$, $\mathrm{E}_{100}, \mathrm{P}_{10}, \mathrm{M}_{100}, \mathrm{~B}_{25}, \mathrm{Rb}_{10}, \mathrm{~Pa}_{100}, \mathrm{~L}_{100}$. The control and treatment regimens each had five mice in Experiment 2, each separate from the mice used for CFU counts in the companion report. The control and treatment regimens each had six mice in Experiment 3.

\section{Tissue collection}

Mice were euthanized the day after the final treatment dose one at a time via $\mathrm{CO}_{2}$ asphyxiation. Upper right lung lobes were flash frozen in liquid nitrogen for immediate RNA preservation then homogenized and lysed via beadbeating as described in Supplemental Material. Remining lung lobes were collected for enumeration of CFU.

\section{Quantification of PD markers}


Following RNA extraction and reverse transcription to cDNA, TaqMan qPCR was used

135 to quantify abundance of $16 \mathrm{~S}$ rRNA as described in Supplemental Material. The RS Ratio was

136 determined in a duplex assay using the QX100 Droplet Digital PCR system (Bio-Rad) as

137 described in Supplemental Material. Primers and probe sequences are in Supplemental Material.

138 CFU burdens were estimated by serial dilutions of lung homogenates and plating on 7H11-

139 OADC agar using $0.4 \%$ activated charcoal to prevent drug carry-over as described in

140 Supplemental Material.

141 Ethical approval and oversight

142 Murine experiments were performed in certified animal biosafety level III facilities with

143 appropriate institutional approvals as described in Supplemental Material.

\section{Statistical analysis}

145 Two-sample Wilcoxon tests were used for pairwise comparisons. For Experiments 2 and

146 3, a Bayesian sigmoidal $\mathrm{E}_{\max }$ model was applied using the function "stan_emax" in the

147 rstanemax $\mathrm{R}$ package to determine, for individual regimens, the treatment duration that results in

$14895 \%$ cure $\left(\mathrm{T}_{95}\right)$. Then, $\mathrm{T}_{95}$ values were compared to establish a rank order of treatment-

149 shortening activity. Lower $\mathrm{T}_{95}$ values indicate greater treatment-shortening activity. Description

150 of the sigmoidal $\mathrm{E}_{\max }$ model is included in Supplemental Material.

$151 \quad$ Hierarchical clustering was used to evaluate the ability of combinations of PD markers to

152 distinguish drugs and regimens. Differences were considered significant at the $95 \%$ confidence

153 level. Analysis was conducted using R (v 3.5.3; R Development Core Team, Vienna, Austria).

156 RS Ratio, CFU, and 16S rRNA burden each measure different biological responses 
Treatment with individual drugs affected each of the three PD markers differently (Fig.

158 1a-c), suggesting that each PD marker measures distinct biological responses. For example,

159 rifampin and isoniazid had similar effects on CFU $(P=0.46)$ but rifampin suppressed the RS

160 Ratio 6-fold more than isoniazid $(P=0.0003)$. Conversely, isoniazid suppressed 16S rRNA

161 burden 25 -fold more than rifampin $(P=0.0003)$. Although both CFU and 16S rRNA burden aim

162 to enumerate the quantity of $M t b$, they did not provide identical information. For example, the

163 effects of isoniazid and bedaquiline on $16 \mathrm{~S}$ rRNA burden were indistinguishable $(P=0.3)$, but

164 bedaquiline reduced CFU 400-fold more than isoniazid $(P=0.001)$. $\log _{10}$ decreases and $P$-values

165 for all drugs and all PD markers are included in Supplemental Material Table S1.

166 Each PD marker assessed the rank order of drugs effect differently. For example,

167 isoniazid had the second greatest effect on both CFU and 16S rRNA burden (Fig. 1b-c) but tied

168 with streptomycin for the least effect on the RS Ratio (Fig. 1a). Pyrazinamide tied with

169 ethambutol for the least effect on CFU (Fig. 1b) but had the third greatest effect on 16S rRNA

170 burden (Fig. 1c) and the RS Ratio (Fig. 1a).

171 Pairwise combinations of PD markers that include the RS ratio distinguish between drugs

172 better than any individual PD marker

173 Although no single PD marker was capable of distinguishing between all individual

174 drugs, the distinct effect of drugs could be resolved based on combinations of PD markers that

175 included the RS Ratio. Hierarchical clustering demonstrated that the combination of the RS Ratio

176 and 16S rRNA burden differentiated each drug from every other drug (Fig. 1d). Similarly, the

177 combination of the RS Ratio and CFU differentiated between all drugs with the exception that

178 streptomycin and ethambutol grouped together (Fig. 1e). By contrast, the combination of CFU

179 and 16S rRNA burden largely failed to distinguish between drugs (Fig. 1f). Only isoniazid and 
180 bedaquiline were clearly distinguishable; other drugs could not be differentiated based on the

181 combination of CFU and 16S rRNA burden.

182 Rank order of treatment-shortening activity in BALB/c relapsing mouse experiments

183 Experiments 2 and 3 quantified treatment-shortening activity of combination drug

184 regimens in the $\mathrm{BALB} / \mathrm{c}$ relapsing TB mouse models based on the conventional microbiologic

185 relapse outcome. The relapse outcomes are summarized in Supplemental Material Table S2.

186 Table 1 summarizes $\mathrm{T}_{95}$ values. In Experiment 2, the rank order of treatment-shortening activity

187 was: $\mathrm{BMZRb}$ (fastest) > BMZ > PMZ > HRZE (slowest) (11). In Experiment 3, the rank order

188 of treatment-shortening activity was: BPaMZ (fastest) $>\mathrm{BPaL}>\mathrm{PaMZ}>\mathrm{HRZE}$ ( (lowest). The

189 sigmoidal $\mathrm{E}_{\max }$ model improved model fit and detected significant differences between treatment

190 regimens compared to the hyperbolic $\mathrm{E}_{\max }$ model $(\gamma=1)$ (Supplemental Material, Fig. S1).

191 Correlation of individual PD markers with treatment-shortening rank order

192 Consistent with Experiment 1, treatment with combination regimens in Experiments 2

193 and 3 affected the RS Ratio, CFU and 16S rRNA burden differently, confirming that they

194 measure distinct biological responses (Fig. 2a-f). Individually, the three PD markers had variable

195 ability to recapitulate the rank order of treatment-shortening activity of regimens (Fig. 3). After

196 only 4 weeks of treatment in Experiment 2, the RS Ratio by itself matched the rank order of

197 treatment-shortening activity measured many months later (Fig. 3a). CFU by itself did not

198 distinguish the first ranked regimen (BMZRb) from the second ranked regimen (BMZ) (Fig. 3b).

199 16S rRNA burden by itself did not match the treatment-shortening rank order except that it

200 distinguished between the second (BMZ) and third (PMZ) ranked regimens (Fig. 3c). After 4

201 weeks of treatment in Experiment 3, the RS Ratio alone did not distinguish between the third

202 (PaMZ) and fourth (HRZE) ranked regimens (Fig. 3d). Likewise, CFU alone did not distinguish 
203 between the second (BPaL) and third (PaMZ) ranked regimens (Fig. 3e). Again, 16S rRNA

204 burden alone largely failed to distinguish between treatment regimens (Fig. 3f). Supplemental

205 Material Table S3 summarizes the correlation of individual PD markers with rank order of

206 regimens at the earliest timepoints for both experiments. Supplemental Material Table S4

207 includes $\log _{10}$ decreases for all treatment regimens and timepoints.

208 A combination of the RS Ratio and CFU improves distinction and classification of

209 treatment regimens

210 Combining different types of PD markers assisted in distinguishing the distinct effects of

211 different regimens. After 4 weeks of treatment, the combination of the RS Ratio with CFU near-

212 perfectly distinguished between regimens in Experiment 2 (Fig. 4a) and perfectly distinguished

213 between regimens in Experiment 3 (Fig. 4d). The degree to which regimens decreased CFU and

214 RS Ratio appeared concordant with treatment-shortening activity (Fig 4a, 4d). By contrast, the

215 combination of 16S rRNA burden with either the RS Ratio (Fig. 4b, Fig. 4e) or CFU (Fig. 4c,

216 Fig. 4f) failed to distinguish between treatment regimens.

\section{DISCUSSION}

219 Our analysis of three BALB/c mouse experiments, conducted at two different institutions

220 using different infecting strains, demonstrated that the RS Ratio, CFU, and 16S rRNA burden

221 each measure different biological responses to drug treatment. The RS Ratio is a non-culture-

222 based assay that provides orthogonal information and correlates with regimen treatment

223 shortening activity. Combining different PD markers enhanced distinction between treatments,

224 relative to any single marker alone. The combination of the RS Ratio with CFU showed the

225 greatest ability to recapitulate the rank order of regimens, providing proof of concept that 
assessment of regimen treatment-shortening activity within the first weeks of treatment may be

227 possible. Development of an early accurate measure of treatment-shortening activity has

228 potential to transform the design of murine efficacy studies, thereby accelerating evaluation of

229 new more potent regimens.

CFU has been a standard historical marker in murine studies despite widely recognized

231 limitations. Our results reinforce previous evidence that change in lung CFU during the first

232 weeks of treatment in mice does not indicate the treatment-shortening activity (1). Perhaps

233 relatedly, McCune and colleagues in the 1950s $(14,15)$ and more contemporary investigators

234 (16-21) have shown that CFU quantifies only the subset of the Mtb population that is capable of

235 growth on solid media and often does not detect the last remaining viable bacilli that determine

236 the treatment duration necessary to prevent relapse in mice. These limitations highlight the

237 potential value of gathering alternative information from murine drug studies and motivated our

238 development of molecular measures of treatment effect.

rRNA has been proposed as a means of enumerating the entire $M t b$ population, including

240 subpopulations capable and incapable of colony formation on solid media (6). de Knegt et. al.

241 previously described a striking divergence between reduction in CFU and reduction in the

242 Molecular Bacterial Load Assay (MBLA, a measure of rRNA burden) in BALB/c mice. For

243 example, de Knegt found that, after 8-12 weeks of treatment with HRZE, CFU decreased >100-

244 fold more than MBLA. Our current experiments demonstrated a similar disconnect in which

245 CFU decreased more than 16S rRNA burden. It remains unclear whether the sustained high

246 rRNA burden during treatment indicates the presence of a continuing large non-culturable $M t b$

247 population or detection of residual rRNA from dead $M t b$. Our experiments confirmed de Knegt's 
observation that change in rRNA burden largely fails to distinguish between regimens with different treatment-shortening potency in mice. measure an alternative property: the degree to which drugs and regimens interrupt rRNA

252 synthesis. Each of our three experiments demonstrated that the RS Ratio provides information

253 that is orthogonal to CFU or rRNA burden. Experiment 2 showed that the RS Ratio was able to 254 measure the effect of adding single drug $(\mathrm{Rb})$ to a potent combination (BMZ), a difference that 255 was not identifiable based on CFU. Change in the RS Ratio correlated with the treatment256 shortening activity of regimens. These observations suggest that the RS Ratio may be a valuable 257 new non-culture-based tool for preclinical efficacy evaluation.

260 PD marker alone. A next step will be development of a composite outcome incorporating CFU

261 and the RS Ratio to improve early efficacy assessment in mice. This would require a

262 development phase in which both CFU and the RS Ratio are collected in additional relapsing

263 mouse trials testing diverse regimens. These results would enable parameterization of a

264 composite outcome and evaluation of the composite CFU-RS Ratio (quantified during the first

265 treatment weeks of treatment) as a surrogate for subsequent relapse. If prediction of relapse is

266 validated, a composite CFU-RS Ratio assay would enable higher throughput murine screening

267 studies in which a large number of regimens is tested in one-month studies to "funnel down" to

268 top candidates that can then proceed to traditional, lengthy, resource intensive, relapsing TB

269 mouse model experiments. Availability of a method that reliably predicts treatment-shortening 
270 efficacy based on responses during the first weeks of treatment would alleviate a key bottleneck

271 in the preclinical TB drug evaluation process.

272 This study has several limitations. First, an inherent challenge to evaluating the predictive

273 value of PD markers in mice is that mice can only be sacrificed once. Because it is not possible

274 to measure PD markers early in treatment and the relapse outcome in the same individual mouse,

275 predictive modeling is inherently limited. Second, as noted above, this report demonstrates proof

276 of concept based on two relapsing mouse studies, establishing a starting point. Parameterization

277 and validation of a composite CFU-RS Ratio will require additional relapse studies with more

278 diverse regimens.

279 In summary, this analysis highlights the potential to harness multiple different types of

280 PD markers to extract greater insight from animal models and accelerate development of new

281 combination regimens. New molecular tools like the RS Ratio offer potential for a new era in

282 which antimicrobial therapies are evaluated not only on culture-based measures of bacterial

283 burden but also on molecular assays that indicate how drugs impact the physiological state of the

284 pathogen.

285

286 SOURCE OF FUNDING

287 The Johns Hopkins study was funded by the U.S. Centers for Disease Control and Prevention's

288 Antibiotic Resistance Solutions Initiative. Otsuka Pharmaceutical donated delamanid and OPC-

289 167832. N.D.W., R.S., and P.N. acknowledge funding from the US National Institutes of Health

290 (1R01AI127300-01A1). N.D.W. and M.I.V. acknowledge funding from the US National

291 Institutes of Health (1R21AI135652-01) and the University of Colorado Department of Medicine 
292 Team Science Award. N.D.W., M.I.V., G.T.R., P.N., and R.S. acknowledge funding from the

293 Bill \& Melinda Gates Foundation (OPP1213947). N.D.W. acknowledges funding from Veterans

294 Affairs (1IK2CX000914-01A1 and 1I01BX004527-01A1). R.S acknowledges funding from the

295 US National Institutes of Health (R01AI135124). P.N. acknowledges funding from the US

296 National Institutes of Health (5R01AI127300).

297

\section{CONFLICT OF INTEREST}

299 The authors declared no conflict of interest.

300

301 DATA AVAILABILTY

302 All primary data is included in the Supplemental Material. 
$\mathbf{a}$
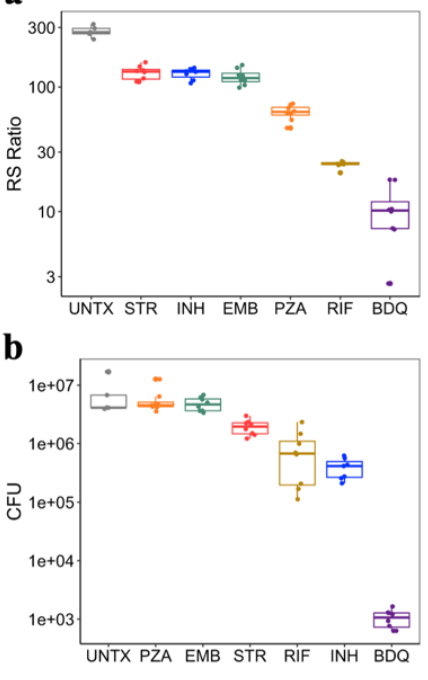

c

UNTX STR RIF EMB PŻA INH BDQ d
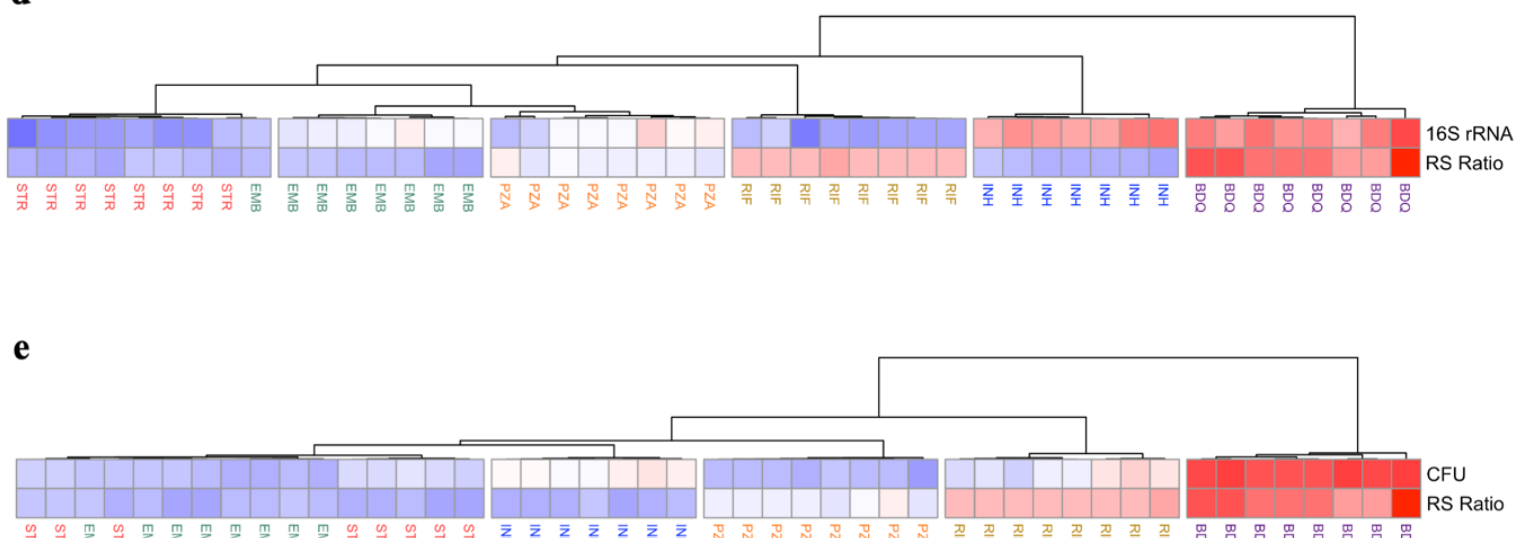

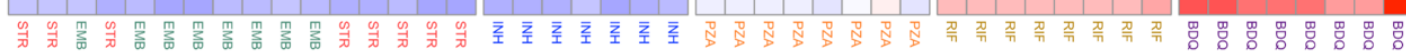

f

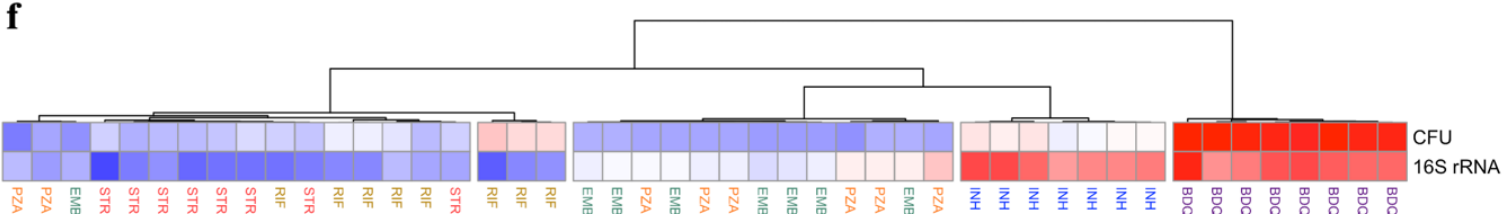

smaller decrease

greater decrease

Figure 1. Effect of 4 weeks of treatment 5 of 7 days per week with individual drugs on different PD markers in the BALB/c mouse high-dose aerosol model. Individual drugs had differing effects on (a) the RS Ratio, (b) CFU and (c) 16S rRNA burden. Points and boxes represent untreated control (gray), streptomycin (red), isoniazid (blue), ethambutol (green), pyrazinamide (orange), rifampin (golden) and bedaquiline (purple). Error bars indicate standard deviations. Hierarchical clustering show drug effect on (d) 16S rRNA burden and the RS Ratio, (e) CFU and the RS Ratio, and (f) CFU and 16S rRNA burden in BALB/c mice. Hierarchical clustering was performed using pheatmap in R with the "Ward.D" agglomeration and "Euclidean" distance methods. Rows represent individual PD markers. Columns represent individual mice. Cell values represent $\log _{10}$ decrease relative to control. Red, white and blue colors indicate greater, average and smaller decrease, respectively. $\mathrm{N}=8$ mice in each treatment group except for untreated control ( $\mathrm{N}=5)$. One mouse in INH group was euthanized (Day 18) due to clinical disease resulting in its removal from the analysis. 
313 Table 1. Treatment-shortening activity of diverse regimens in two BALB/c TB relapsing mouse model experiments. Regimen composition, $\mathrm{T}_{95}$ and rank order of treatment-shortening activity are shown for Experiments 2 and 3.

\begin{tabular}{|c|c|c|}
\hline Experiment and regimen & $\begin{array}{c}\mathrm{T}_{95} \text { in weeks } \\
\text { [95\% confidence interval] }\end{array}$ & $\begin{array}{c}\text { Rank of } \\
\text { treatment-shortening activity }\end{array}$ \\
\hline \multicolumn{3}{|l|}{ Experiment 2} \\
\hline bedaquiline, moxifloxacin, pyrazinamide, rifabutin (BMZRb) & $6.10[5.68,6.31]$ & 1 \\
\hline bedaquiline, moxifloxacin, pyrazinamide (BMZ) & $7.09[7.05,7.28]$ & 2 \\
\hline rifapentine, moxifloxacin, pyrazinamide (PMZ) & $8.03[7.64,9.82]$ & 3 \\
\hline isoniazid, rifampin, pyrazinamide, ethambutol (HRZE) & $18.63[18.44,18.90]$ & 4 \\
\hline \multicolumn{3}{|l|}{ Experiment 3} \\
\hline bedaquiline, pretomanid, moxifloxacin, pyrazinamide (BPaMZ) & $5.59[5.33,6.13]$ & 1 \\
\hline bedaquiline, pretomanid, linezolid (BPaL) & $10.00[9.79,10.30]$ & 2 \\
\hline Pretomanid, moxifloxacin, pyrazinamide (PaMZ) & $13.12[12.06,13.68]$ & 3 \\
\hline isoniazid, rifampin, pyrazinamide, ethambutol (HRZE) & $21.21[20.70,21.78]$ & 4 \\
\hline
\end{tabular}



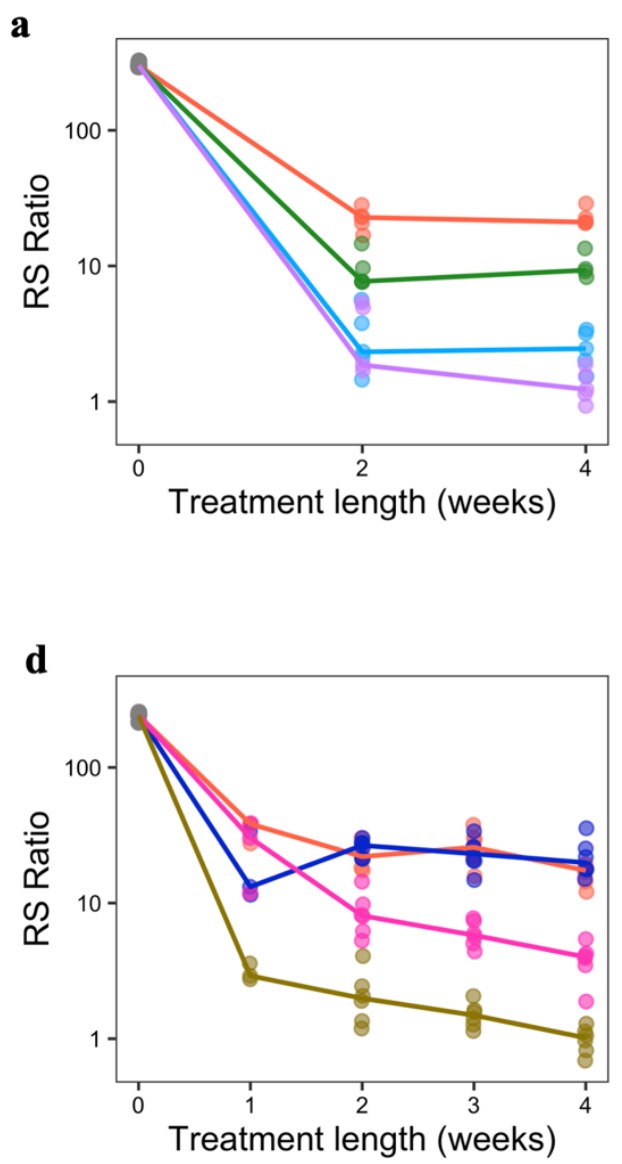
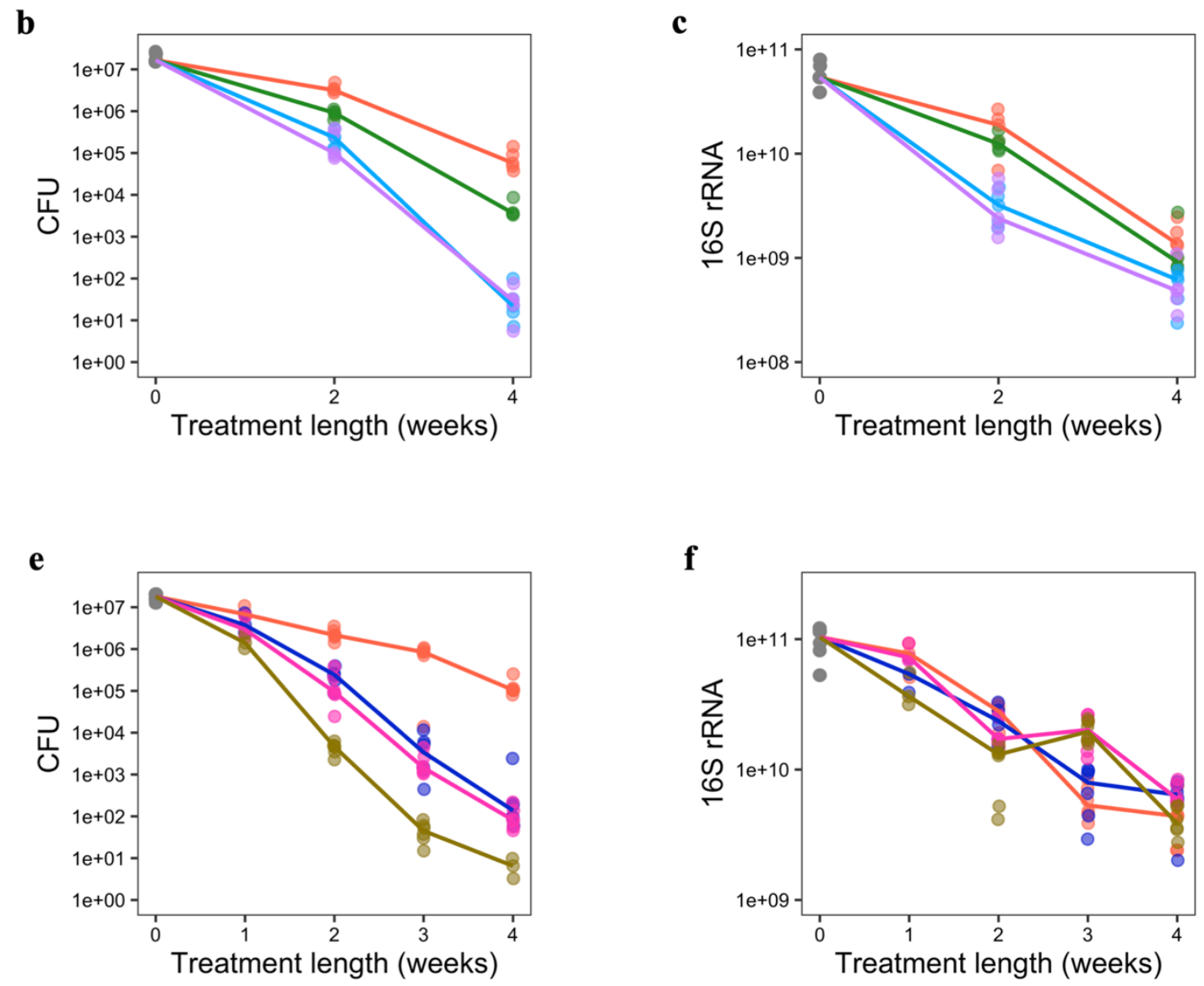

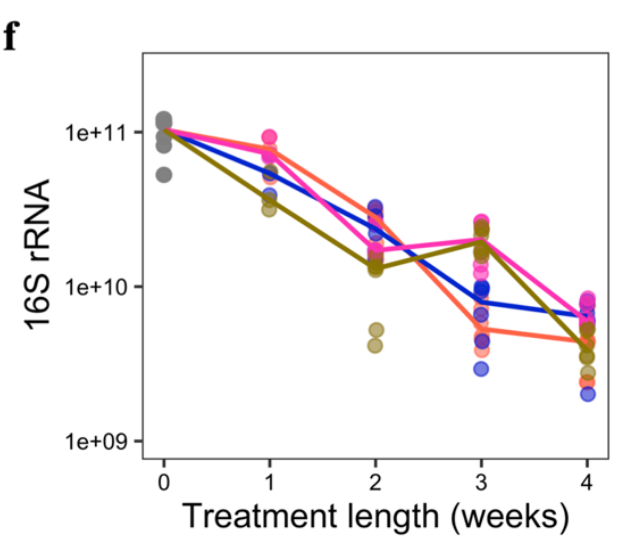

Figure 2. Differing effects of treatment on three PD markers during the first 4 weeks of treatment using data from two BALB/c TB relapsing mouse model experiments. RS Ratio, CFU and 16S rRNA burden were measured in lung homogenate of untreated mice (grey) and mice treated with BMZRb (purple), BMZ (light blue), PMZ (green) and HRZE (orange) in Experiment 2 (a, b, c), and with BPaMZ (golden), BPaL (pink), PaMZ (blue) and HRZE in Experiment 3 (d, e, f). Dots represent values from individual mice. Solid lines connect median values. All graphics use a $\log _{10}$ scale for the $\mathrm{Y}$ axis. The control and treatment regimens each had 5 mice (Experiment 2) and 6 mice (Experiment 3 ). 

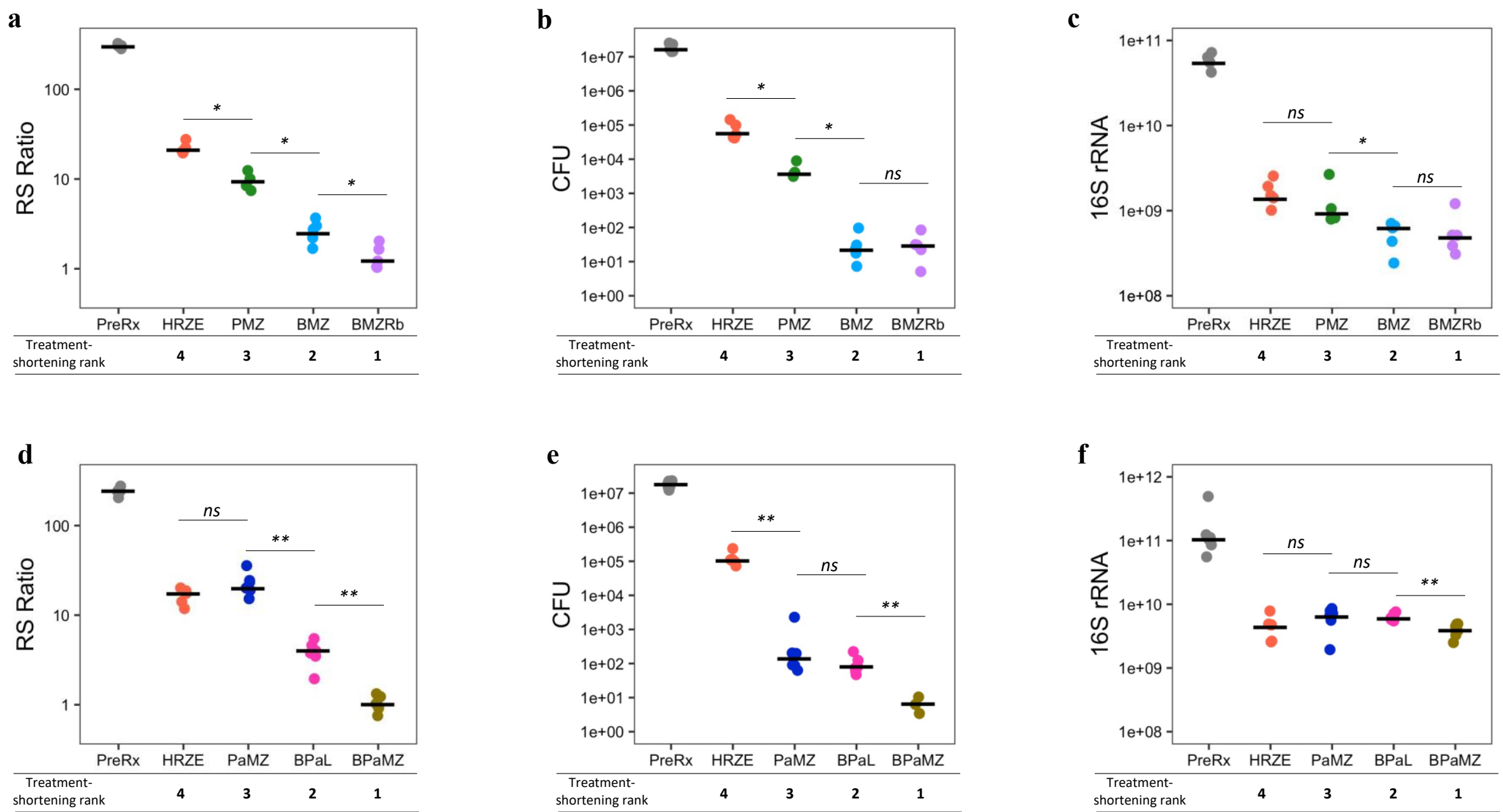

Figure 3. Correlation of RS Ratio, CFU and 16S rRNA burden with treatment-shortening rank order after 4 weeks of treatment using data from two BALB/c TB relapsing mouse model experiments. RS Ratio, CFU and 16S rRNA burden were measured in lung homogenate of untreated mice (grey) and mice treated with BMZRb (purple), BMZ (light blue), PMZ (green) and HRZE (orange) in Experiment 2 (a, b, c), and with BPaMZ (golden), BPaL (pink), PaMZ (blue) and HRZE in Experiment 3 (d, e, f). Dots represent values from individual mice. Bars represent median values. $P$-value symbols are as follows: ns is non-significant, $*$ is $P$-value $<0.05$, 
a

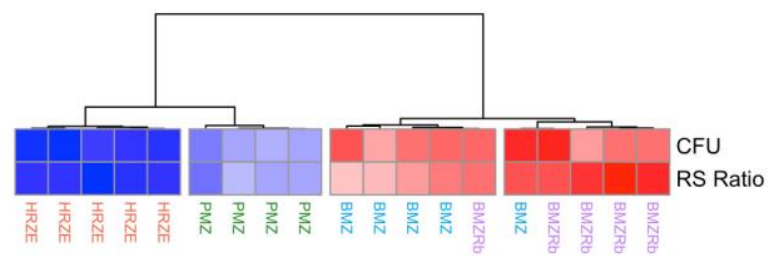

b

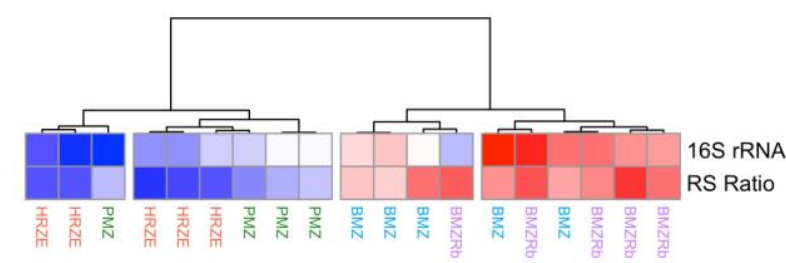

c

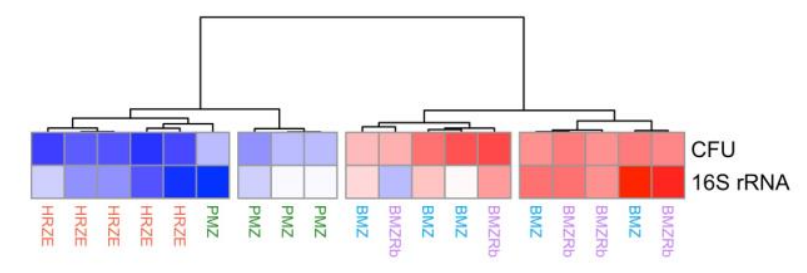

d

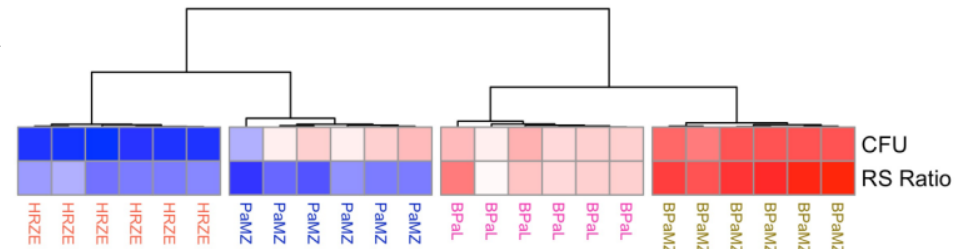

e

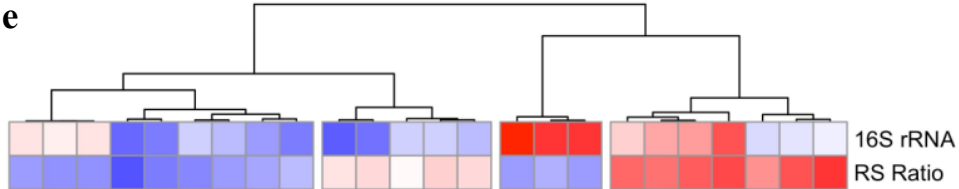

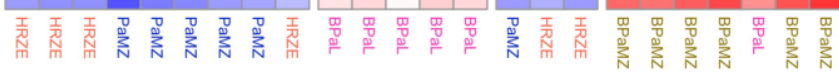

f

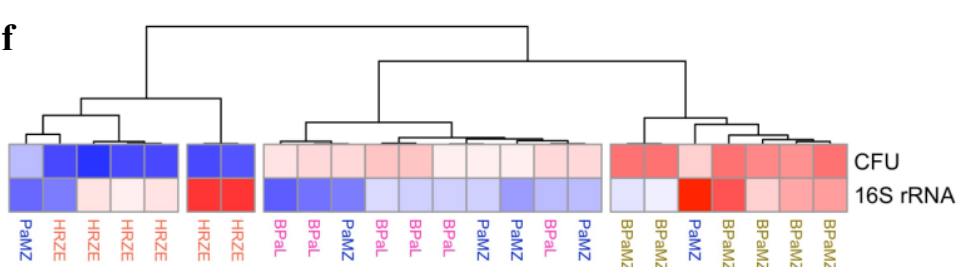

greater decrease

Figure 4. Distinguishing different regimens based on three PD markers measured after 4 weeks of treatment using data from two BALB/c TB relapsing mouse model experiments. Combination of PD markers are shown for Experiment 2 (a, b, c) and Experiment 3 $(\mathbf{d}, \mathbf{e}, \mathbf{f})$. Hierarchical clustering was performed using pheatmap in R with the "Ward.D" agglomeration and "Euclidean" distance methods. Rows represent individual PD markers. Columns represent individual mice. Cell values represent $\log _{10}$ decrease relative to control. Red, white and blue colors indicate greater, average and smaller decrease, respectively. The control and treatment regimens each had 5 mice (Experiment 2) and 6 mice (Experiment 3). RS Ratio could not be quantified in one mouse treated with PMZ in Experiment 2 resulting in its removal from the analysis. 


\section{REFERENCES}

340 1. Gumbo T, Lenaerts AJ, Hanna D, Romero K, Nuermberger E. 2015. Nonclinical models for 341 antituberculosis drug development: a landscape analysis. J Infect Dis 211 Suppl 3:S83-95.

342 2. De Groote MA, Gilliland JC, Wells CL, Brooks EJ, Woolhiser LK, Gruppo V, Peloquin

343 CA, Orme IM, Lenaerts AJ. 2011. Comparative studies evaluating mouse models used for 344 efficacy testing of experimental drugs against Mycobacterium tuberculosis. Antimicrob 345 Agents Chemother 55:1237-1247.

346 3. Zhan L, Tang J, Sun M, Qin C. 2017. Animal models for tuberculosis in translational and 347 precision medicine. Front Microbiol 8:717.

348 4. Honeyborne I, McHugh TD, Phillips PPJ, Bannoo S, Bateson A, Carroll N, Perrin FM, 349 Ronacher K, Wright L, van Helden PD, Walzl G, Gillespie SH. 2011. Molecular bacterial 350 load assay, a culture-free biomarker for rapid and accurate quantification of sputum Mycobacterium tuberculosis bacillary load during treatment. J Clin Microbiol 49:3905the Evaluation of Anti-tuberculosis Antibiotics (PanACEA). 2014. The molecular bacterial load assay replaces solid culture for measuring early bactericidal response to antituberculosis treatment. J Clin Microbiol 52:3064-3067. 
response by colony forming units, time to culture positivity and the molecular bacterial load assay compared in a mouse tuberculosis model. Tuberculosis 105:113-118.

7. Andries K, Gevers T, Lounis N. 2010. Bactericidal potencies of new regimens are not predictive of their sterilizing potencies in a murine model of tuberculosis. Antimicrob Agents Chemother 54:4540-4544.

8. Mitchison DA, Davies GR. 2009. Assessment of the efficacy of new anti-tuberculosis drugs. Open Infect Dis J 2:59-76.

9. Grosset JH, Tyagi S, Almeida DV, Converse PJ, Li S-Y, Ammerman NC, Bishai WR,

Enarson D, Trébucq A. 2013. Assessment of clofazimine activity in a second-line regimen for tuberculosis in mice. Am J Respir Crit Care Med 188:608-612.

10. Walter ND, Born SEM, Robertson GT, Reichlen M, Dide-Agossou C, Ektnitphong VA, Vásquez JJ, Voskuil MI. 2021. Mycobacterium tuberculosis precursor rRNA as a measure 
murine tuberculosis models. Antimicrob Agents Chemother SUBMITTED AS COMPANION REPORT.

12. Kelly BP, Furney SK, Jessen MT, Orme IM. 1996. Low-dose aerosol infection model for testing drugs for efficacy against Mycobacterium tuberculosis. Antimicrob Agents

13. Lenaerts AJ, Gruppo V, Marietta KS, Johnson CM, Driscoll DK, Tompkins NM, Rose JD, Reynolds RC, Orme IM. 2005. Preclinical testing of the nitroimidazopyran PA-824 for activity against Mycobacterium tuberculosis in a series of in vitro and in vivo models.

14. McCune RM, Tompsett R, McDermott W. 1956. The fate of Mycobacterium tuberculosis in mouse tissues as determined by the microbial enumeration technique. II. The conversion of tuberculous infection to the latent state by the administration of pyrazinamide and a

15. McCune RM, Feldmann FM, Lambert HP, McDermott W. 1966. Microbial persistence. I. The capacity of tubercle bacilli to survive sterilization in mouse tissues. J Exp Med companion drug. J Exp Med 104:763-802.

16. Chengalroyen MD, Beukes GM, Gordhan BG, Streicher EM, Churchyard G, Hafner R, Warren R, Otwombe K, Martinson N, Kana BD. 2016. Detection and Quantification of $123: 445-468$. Differentially Culturable Tubercle Bacteria in Sputum from Tuberculosis Patients. Am J Respir Crit Care Med https://doi.org/10.1164/rccm.201604-0769OC. 
17. Mukamolova GV, Turapov O, Malkin J, Woltmann G, Barer MR. 2010. Resuscitationpromoting factors reveal an occult population of tubercle bacilli in sputum. Am J Respir Crit Care Med 181:174-180.

18. Dhillon J, Fourie PB, Mitchison DA. 2014. Persister populations of Mycobacterium tuberculosis in sputum that grow in liquid but not on solid culture media. J Antimicrob Chemother 69:437-440.

19. Saito K, Warrier T, Somersan-Karakaya S, Kaminski L, Mi J, Jiang X, Park S, Shigyo K, polymerase in antibiotic-tolerant Mycobacterium tuberculosis results in differentially

20. McAulay K, Saito K, Warrier T, Walsh KF, Mathurin LD, Royal-Mardi G, Lee MH, Ocheretina O, Pape JW, Fitzgerald DW, Nathan CF. 2018. Differentially detectable Mycobacterium tuberculosis cells in sputum from treatment-naive subjects in Haiti and their proportionate increase after initiation of treatment. mBio 9. in vivo. Front Microbiol 6. 